- Labial artery swelling as a unique presentation of multiple myeloma without osteolytic lesions or any other soft tissue involvement in the mouth is very uncommon.

- This report describes such a case and also discusses diagnosis, treatment options and dental implications of multiple myeloma.

- Provides useful advice for clinicians faced with a similar case.

\title{
Low grade multiple myeloma that presented as a labial swelling - A case report
}

\author{
S. Ariyaratnam, ${ }^{1}$ C. Sweet ${ }^{2}$ and J. Duxbury ${ }^{3}$
}

Myeloma is a disease of the bone marrow in which there is malignant proliferation of plasma cells. Myeloma is usually associated with the accumulation of a monoclonal immunoglobulin or light chains in plasma and subsequently an increase in light chains in the urine (Bence-Jones proteins). Renal failure can occur and bone destruction in the axial skeleton may ensue with pain and fractures. Amyloidosis associated with multiple myeloma is a relatively common finding. The most frequently reported oral sign of amyloidosis is macroglossia. There are numerous cases in the literature of amyloid deposition in tongues related to multiple myeloma. However, none of these cases describe amyloid deposition surrounding arteries in the oral cavity. We therefore report a case of an innocuous lower labial swelling, which subsequently led to the diagnosis of amyloid deposition surrounding an artery, the cause of which was later shown to be multiple myeloma.

\section{CASE REPORT}

A 77-year-old lady was referred to the oral medicine unit at the University Dental Hospital of Manchester by her general dental practitioner, regarding swellings on the patient's lower lip and cheek. The patient complained of raised lesions in the lower lip and left buccal mucosa that had been present for six months and one year respectively. No change in size or consistency had been noted over this period. The lesions were asymptomatic and were thought to have been caused by trauma while chewing.

\footnotetext{
1*S. Ariyaratnam, Specialist in Oral Medicine and Surgical Dentistry, Unit of Oral Medicine, University Dental Hospital of Manchester, Manchester, M15 6FH; ${ }^{2} \mathrm{C}$. Sweet, Senior House Officer, University Dental Hospital of Manchester, Higher Cambridge Street, Manchester, M15 $6 \mathrm{FH}^{3}{ }^{3}$. Duxbury, Consultant in Oral Medicine, Honorary Senior Lecturer, Unit of Oral Medicine, University Dental Hospital of Manchester, Manchester, M15 6FH. ${ }^{*}$ Correspondence to: Dr Senathirajah Ariyaratnam Email:senathirajah.ariyaratnam@man.ac.uk
}

\section{Refereed Paper}

Accepted 28 January 05

doi: $10.1038 /$ sj.bdj. 4812742

๑ British Dental Journal 2005; 199: 433-435
Medically the patient suffered occasional migraine-type headaches and had an episode of vertigo six months previously. She had undergone a hysterectomy 22 years previously, was a non-smoker, occasionally drank alcohol but was otherwise fit and well.

Extra-orally no swellings or abnormalities were evident. Intra-orally a firm rubbery swelling of approximately $10 \mathrm{~mm}$ diameter was present in the left buccal mucosa, adjacent to the UL4 (24) and a lesion of similar consistency was present in the lower labial mucosa. The latter was cigar shaped, approximately $20 \mathrm{~mm}$ in length and $5 \mathrm{~mm}$ wide.

A differential diagnosis of lymphangiomous or lipomatous deposits was made. Arrangements were made for blood tests including plasma cholesterol and for biopsy. The haematology results showed a normal white cell count, but mild anaemia with normal cholesterol level.

At the biopsy appointment prior aspiration of the lesion showed pulsating blood, which suggested the lesion was arterial in origin. Closer inspection revealed a similar lesion in the upper labial mucosa, vascular enlargement on the ventral surface of the tongue and prominence and firmness of the left temporal artery.

Calcification of these arteries was suspected so the patient was referred to their general medical practitioner (GMP) with a view to seeking the opinion of a vascular specialist. However at a later review it was discovered the patient had made a private self-referral to a consultant ENT surgeon with no subsequent definitive outcome.

The patient also now reported feeling generally tired. In view of this delay in obtaining a diagnosis the patient was referred to a consultant in oral and maxillofacial surgery at the Manchester Royal Infirmary for biopsy of the vascular lower lip lesion. Histopathological examination revealed a blood vessel surrounded by gross deposits of hyaline eosinophilic material. The material was strongly stained by congo red and apple-green birefringences were present between crossed polarised light. The features of this material were consistent with amyloid deposits.

Referral was made to a consultant haematologist who undertook blood tests and urinalysis, which indicated the presence of paraproteinaemia and Bence-Jones 
protein in the urine. Subsequent bone marrow studies provided clear evidence of a diagnosis of multiple myeloma, although a radiographic examination revealed no osteolytic lesions. Renal function was found to be normal. The myeloma was classified as monoclonal gammopathy of undetermined significance (MGUS) and as such no active treatment was planned other than regular haematological review.

\section{DISCUSSION}

\section{Multiple myeloma}

There are approximately 3,000 new cases of myeloma reported in the UK each year representing 15\% of blood cancers and $1 \%$ of all types of cancer. It is more common in males, with a male:female ratio of $3: 2$. The incidence rises with age. ${ }^{1-3}$ In multiple myeloma a diffuse neoplastic proliferation of plasma cells which produce immunoglobulin is seen throughout the red marrow. This uncontrolled growth of plasma cells has many consequences including skeletal destruction, bone marrow failure, increased plasma volume and viscosity, suppression of normal immunoglobulin production and renal insufficiency. ${ }^{3}$ Osteolytic lesions are common in the jaws and may be the first sign of disease, presenting clinically as a generalised osteoporosis or as discrete, wellcircumscribed, 'punched-out' radiolucent areas on radiographic examination. ${ }^{4}$ Classically a lateral skull radiograph shows this 'punched-out' appearance. These areas occur as a result of neoplastic plasma cells producing osteoclast-activating factor. The mandible is more commonly affected than the maxilla, with the premolar area, molar area and ramus being the most frequently reported sites. In some cases asymptomatic lesions may be identified as a result of routine dental radiographs, particularly on panoramic films. ${ }^{4}$ Cases of misdiagnosed periapical lesions may lead to endodontic reatment being carried out on what is believed to be a non-vital tooth. ${ }^{4}$ In one case reported, the initial symptoms were pain and dysfunction of the temporomandibular joint that resulted from pathological fracture of the mandibular condyle. ${ }^{5}$

Some lesions cause intraosseous expansion and compression resulting in paraesthesia, pain, swelling and pathological fracture. ${ }^{4}$ Lesions may also involve the oral soft tissues and appear as tumours of the gingiva, maxillary tuberosity, or alveolar mucosa. ${ }^{4}$

The plasma cells in multiple myeloma secrete a monoclonal immunoglobulin, a paraprotein, which forms a discrete band on electrophoresis. This is most commonly of the IgG subtype, but production tends to be asynchronous which leads to incom- plete immunoglobulin molecules and an excess of light chains of the Kappa or Lambda type. Whole immunoglobulin molecules cannot pass into urine through the glomerular filtration, but the light chains can. When present in the urine they are known as Bence-Jones proteins and are detectable on urinalysis. The plasma concentration of normal immunoglobulins is often reduced; this is known as immune paresis and results in frequent infections.

Bone pain, especially back pain is a major symptom reported by sufferers. Sudden severe pain occuring without trauma may be associated with vertebral collapse or spontaneous fracture. ${ }^{3}$ A deterioration in general health is usually noted and it is not uncommon for the patient to complain of the symptoms of fatigue, weakness and malaise. Spinal cord compression can occur, leading to neurological symptoms.

Hypercalcaemia can result from increased bone destruction. Anaemia is common and a raised erythrocyte sedimentation rate (ESR) may be noted on haematological investigation. ${ }^{3}$ Renal failure may ensue leading to further haematological abnormalities and the symptoms of anorexia, depression and vomiting. A subsequent loss of water and electrolytes leads to dehydration and can exacerbate any renal disease present. Ultimately multiple myeloma leads to death.

\section{Amyloid}

Amyloidosis refers to the extracellular deposition of any group of unrelated proteins. ${ }^{6}$ With light microscopy, amyloid appears as an eosinophilic amorphous substance, which on congo red staining demonstrates green birefringence when polarised light is used. ${ }^{6,7}$ Amyloidosis occurs in 5-15\% of patients with multiple myeloma. ${ }^{4}$ Immunoglobulin light chains are the precursor proteins for fibrils that are formed during light chain type amyloidosis (AL). ${ }^{8-10}$ This is the only type of amyloidosis associated with multiple myeloma. ${ }^{10}$ As levels of light chains increase they may cause amyloid deposition. The deposits may be systemic (ie distributed throughout many organs of the body) or localised (ie restricted to single organs such as the lungs, brain, or skin). ${ }^{6}$

Most noticeably for the dentist, amyloidosis can affect the mucous membranes of the oral cavity. When this occurs it predominantly involves the tongue resulting in macroglossia. ${ }^{6}$ Amyloidosis may also affect the salivary glands leading to enlargement and decreased salivary flow. Discrete yellow nodules affecting the tongue, palate, buccal and labial mucosa are recognised clinical presentations. ${ }^{4}$
Amyloid is found most frequently around blood vessels and muscle tissue, ${ }^{4}$ however there are no cases reported in the recent literature of amyloid surrounding arteries in the oral cavity.

\section{Classification and treatment of multiple myeloma}

Prognosis in myeloma is determined by both the number and specific properties of myeloma cells in a given patient. These specific properties include growth rate, production rate of monoclonal proteins and production or non production of various cytokines and chemicals which damage or significantly impair other tissue, organ or bodily functions. ${ }^{3}$

The Durie-Salmon(DS) clinical staging system is a commonly used staging system for multiple myeloma. ${ }^{11}$ This system brings together the major clinical parameters in correlation with measured myeloma cell mass (the total number of myeloma cells in the body). ${ }^{11}$ There are three staging categories: I, II and III. This staging makes it possible to categorise patients and select optimal therapy. ${ }^{3}$ The DS staging system was developed in the mid 1970s and has been used successfully since this time for clinical trial research. Other systems have been developed but have yet to be accepted as better alternatives. ${ }^{12}$ Since 1975 several new parameters have been discovered. Among these are serum B2 microglobulin, a measure of disease activity; as well as labelling index (L1\%), a specific indicator of growth rate. In the early 1990s Durie presented a variation of the scheme which uses these parameters. ${ }^{13}$

Multiple myeloma is not curable; the first and most important decision is to ascertain whether treatment is required. Patients with monoclonal gammopathy of undetermined significance (MGUS) and smouldering myeloma should be observed closely rather than treated. ${ }^{14}$

Since melphalan was introduced in the early 1960s various combination chemotherapy regimens have been used and attempts made to improve outcomes using high dose chemotherapy regimens with bone marrow transplant or stem cell rescue. There is as yet no consensus as to the best way to manage multiple myeloma. ${ }^{3,15}$ A median survival rate of three years has been reported in the literature when using a regimen of melphalan and prednisolone. ${ }^{14}$ However in the last 15 years there has been dramatic modification in the management of multiple myeloma. Front line therapy for younger patients now involves high-dose therapy with autologous stem cell transplantation with increased survival rates being reported. ${ }^{14}$

It has been noted that bisphosphonates reduce the incident of skeletal-related 
events recombinant erythropoietin reduces red blood cell transfusion need, both of which improve quality of life. Thalidomide alone or in combination with dexamethasone has shown promising results and is being evaluated for front line treatment. ${ }^{16}$

Treatment is recommended when the monoclonal protein increases and/or clinical problems have emerged or are imminent. Problems sufficient to require treatment include bone destruction, renal insufficiency, reduced blood counts, elevated blood calcium, nerve damage, or other significant organ or tissue damage. The overall goals of treatment are to address specific problems and to achieve general control of the disease.

\section{Dental complications}

There are a number of concerns for the general dental practitioner with regards to treating a patient with multiple myeloma. Bleeding and infection require careful consideration. Bleeding tendencies occur as a result of thrombocytopenia and from high levels of the abnormal circulating proteins, which interfere with coagulation. ${ }^{4}$ Renal failure may also occur as a result of excessive serum proteins. Susceptibility to infection increases as immune paresis occurs, and the level of circulating, normal immunoglobulins, decreases. If a patient is to undergo dental treatment it is vital to determine their current renal status and medication. Chemotherapy and steroid therapy affect the patient's bone marrow and adrenal function respectively. Elective dental treatment should be avoided and ideally be performed when the patient is in remission.

A clinician who is faced with a swelling similar to the two described in this case report should ensure that a thorough history and clinical examination is carried out. A dentally qualified clinician may appropriately examine other superficial branches of the carotid artery. It is important that if the lesion is thought to be vascular in origin the clinician should not simply excise it. The result of this could be severe haemorrhaging, both difficult to control and disconcerting for patient and clinician. Swellings may be palpated for a pulse, which would indicate a lesion to be associated with an artery. Aspiration prior to biopsy may be carried out using a fine bore needle. Lesions associated with arteries spurt with blood, while blood from veins tends to flow. Final confirmation of the cause of a swelling is by biopsy. But referral to a specialist should be made for biopsy related to an arterial lesion because of the risk of haemorrhage.

This case illustrates a rare and unusual presentation of multiple myeloma, with very few similar documented cases of labial artery involvement reported in the literature. However, it is important that clinicians are aware that such a potentially life-threatening disorder can present in such an innocuous way. Likewise the clinican should have an understanding of the most appropriate way to deal with such a case.
1. Reidel D A, Potten L M, Blattner W A. Epidemiology of multiple myeloma. In Wiernik P H, Canellos G P, Kyle P A, Schiffer C A (Eds) Neoplastic diseases of the blood. Vol 1, $2^{\text {nd }}$ ed. pp 347-372. New York, Edinburgh, London, Melbourne, Tokyo: Churchill Livingstone, 1991.

2. Barlogie B, Epstein J, Selvanayagam P, Alexanian R Plasma cell myeloma: New biological insights and advances in therapy. Blood 1989; 73: 965-978.

3. Durie B G M. Multiple myeloma. A concise review of the disease and treatment options. UK: International Myeloma Foundation, Nov 2000

4. Rose L F, Kaye D. Internal medicine for dentistry. $2^{\text {nd }}$ Ed. pp 366-367. UK: CV Mosby, 1990.

5. Jagger R G, Helkimo M, Carlsson G E. Multiple myeloma involving the temoromandibular joint. J Oral Surg 1978; 36: 557-559.

6. Breathnach S M. Amyloid and amyloidosis. J Am Acad Dermatol 1988; 18: 1-16.

7. Touart D M, Sau P. Cutaneous deposition diseases. Part 1. J Am Acad Dermatol 1998; 39: 149-171.

8. Stevens FJ, Kisilevsky R. Immunoglobulin ligh chains, glycosaminoglycans, and amyloid. Cell Mol Life Sci 2000; 3: 441-449.

9. Van Der Waal R I F, Van De Scheur M R, Huijgens P C Starink T M, Van De Waal I. Amyloidosis of the tongue as a paraneoplastic marker of plasma cell dyscrasia. Oral Surg Oral Med Pathol Oral Radiol Endod 2002; 94: 444-447.

10. Gretz M A, Lacy M Q, Dispenzieri A. Amyloidosis. Haematol Oncol Clin North Am 1999; 13: 1211-1233.

11. Durie B G M, Salmon S E. A clinical staging system for multiple myeloma. Cancer 1975; 36: 842-854.

12. Jacobson J L, Hussein M A, Barlogie B, Durie B G M, Crowley J L. A new staging system for multiple myeloma patients based on the southwest oncology group (SWOG) experience. Br J Haemato/ 2003; 122: 441

13. Durie B G M, Stock-Novack D, Salmon SE et al. Prognostic value of pre treatment serum B2 microglobulin in myeloma: A South West oncology group study. Blood 1990; 75: 823-830.

14. Kyle R A. 'Benign' monoclonal gammopathy - after 20-35 years follow up. Mayo Clinic Proceedings 1993; 68: 26-36.

15. Alexanian R, Haut A, Khan A et al. Treatment for multiple myeloma: combination chemotherapy with different melphalan dose regimes. J Am Med Asso 1969; 208: 1680-1685.

16. Harousseau J L. Management of multiple myeloma. Rev Clin Exp Haematol 2002; 6: 253-275. 